\title{
La real expedición filantrópica de la vacuna (Xavier de Balmis/Josep Salvany). 1803-1806
}

\author{
Francesc Asensi Botet
}

\section{Hospital Universitari La Fe. Valencia, España}

Recibido: 24 de agosto 2009 Aceptado: 8 de Septiembre 2009

Correspondencia a:

Francesc Asensi Botet asensifrancesc@gmail.com

\section{The royal philanthropic expedition of the vaccine (Xavier de Balmis/Josep Salvany). 1803-1806}

Six years after Jenner discovered the anti-smallpox vaccine, King Charles IV mandated the initiation of vaccination campaigns throughout his kingdom. The overseas campaign was coordinated by the Valencian military doctor Xavier de Balmis with the aid of the Catalan Josep Salvany. The vaccine was transported to America "in vivo" in 22 children. The expedition departed from La Coruña on November 1806 and arrived to the Venezuelan port of La Guayra where it was split in two: One sub expedition under the order of Josep Salvany, continued by land to Colombia, Ecuador, Peru and Bolivia. Salvany died in the Bolivian city of Cochabamba. The other sub expedition, leaded by Balmis himself, continued by sea to Cuba and Mexico. From Acapulco it arrived to Manila and from there to Macau followed by inland penetration into China.

Key words: Smallpox, vaccine, history.

Palabras clave: Viruela, vacuna, historia.

$\mathrm{U}$ na vez prácticamente superadas, con sus luces y sus sombras, las fases de conquista y colonización de las tierras americanas durante los siglos XVI y XVII, las monarquías europeas, y en particular la hispánica, organizaron y subvencionaron durante los siglos XVIII y XIX una serie de expediciones de carácter científico con objeto de estudiar la geografía, la flora, la fauna, la etnografía, la lingüística, etc. Estas expediciones proporcionaron una ingente cantidad de información que enriqueció de forma decisiva nuestros conocimientos en todos los campos de la Ciencia. Entre estas expediciones se encuentran algunas de trascendental importancia como la dirigida por Jorge Juan para medir la "hinchazón" de la Tierra en el ecuador, cuyos principales trabajos se realizaron en Guayaquil y sus alrededores, la de José Celestino Mutis para el estudio de la flora de "Nuevo Mundo", la de Alexander von Humboldt que hizo importantísimos descubrimientos geográficos y naturalistas justamente calificado de "segundo descubridor de América", y últimamente la de Charles Darwin cuya importancia en biología evolutiva no es necesario resaltar en este segundo centenario de su nacimiento.

Perfectamente equiparable a estas grandiosas expediciones fue otra cuyo objetivo, a diferencia de ellas, no fue explorar ni descubrir nada sino aplicar un reciente descubrimiento en beneficio de las masas de población de las colonias españolas que se veían azotadas por una epidemia mortífera traída a estas tierras por los coloni- zadores. De alguna manera se trataba de paliar el daño que se les había hecho. Esta expedición, calificada muy justificadamente de "filantrópica" (el nombre oficial fue "Real Expedición Filantrópica de la Vacuna"), dirigida por Xavier de Balmis es el tema que nos va a ocupar.

La viruela era una enfermedad infecciosa sumamente contagiosa con una mortalidad que llegaba al $20 \%$. Entre los supervivientes las secuelas eran muy graves incluyendo la ceguera en $\sim 30 \%$. Conocida desde la más remota antigüedad en las civilizaciones asiria, babilónica, egipcia y china llegó más tarde a Europa donde se propagó provocando grandes epidemias durante los siglos XVI a XVIII. Se calcula que a finales del siglo XVIII morían anualmente por viruela en Europa unas 400.000 personas.

Parece ser que la viruela fue introducida en América en 1520 por un esclavo negro de Pánfilo Narváez cuando perseguía las tropas de Hernán Cortés. En el siglo XVII la viruela se había extendió ya por toda América convirtiéndose en una auténtica enfermedad social, particularmente en las zonas de mayor actividad comercial como el Caribe (Figura 1). Hubo dos grandes epidemias en 1780 y 1798. Fue calificada por Timoteo O'Scanlan de "guadaña que siega sin distinción la cuarta parte del género humano" y el mismo autor, ante los éxitos de la inoculación, auguró: " podemos tener esperanza algún día de lograr artificialmente su exterminio".

Todos los intentos terapéuticos resultaron vanos lo que aumentó el interés por su prevención. Empíricamente se utilizó la llamada "variolización" consistente en la 
inoculación cutánea del líquido procedente de la vesícula de un enfermo. Con ello se provocaba una forma leve de viruela que no dejaba de tener efectos patógenos y con una mortalidad diez veces menor que la infección natural. Lo que se estaba haciendo sin saberlo era una especie de vacunación transmitiendo la infección por una vía, la cutánea, diferente a la de propagación natural de la enfermedad que era la vía respiratoria.

La introducción de esta técnica no estuvo exenta de polémica. En una primera etapa predominó el rechazo, incluso por parte de las autoridades eclesiásticas que veían en ella una intromisión ilícita en los designios divinos (i!). Tras acaloradas polémicas predominó la aceptación y difusión. En España empezó a practicarse al mismo tiempo que en América. Un misionero carmelita observó los éxitos y empezó a practicar la variolización entre sus fieles de Pará en Brasil. En 1777 se introdujo en Buenos Aires, posteriormente en Lima, Santa Fe de Bogotá y a finales de siglo en México.

Como es bien sabido, un enorme paso en la lucha contra la viruela es el dado por el médico rural inglés Edward Jenner cuando observó que quienes se infectaban de la viruela de las vacas, una enfermedad leve y sin secuelas, no padecían la grave viruela humana. Lo comprobó científicamente mediante un ensayo que hoy probablemente le hubiera llevado a la cárcel: infectó a un niño con viruela vacuna adquirida por una ordeñadora de vacas y un tiempo después le inoculó líquido de una lesión de viruela humana y vio que no le ocurría nada (Figura 2). Había dado comienzo una nueva era en la Historia de la Medicina, la era de las vacunas, de una enorme expansión en las últimas décadas. Precisamente esa primera infección contra la que se dispuso de una vacuna ha sido también la primera en haberse declarado en 1980 erradicada del planeta Tierra gracias a aquel primer paso dado por Jenner.

La técnica de Jenner consistía en inyectar en la piel no el líquido de una lesión de un enfermo de viruela humana como en la "variolización" sino el líquido (o "linfa") de la vesícula provocada por la inyección de una lesión de viruela vacuna (Figura 3). Una vez "vacunada" (empezó a utilizarse la palabra) una persona se extraía "linfa" de la vesícula provocada por la "vacunación" para vacunar a otra persona. De esta forma fue transmitiéndose "brazo a brazo" una medida eficazmente protectora frente a la terrible enfermedad de la viruela.

Apenas siete años después de que Edward Jenner introdujera esta vacuna antivarólica la Corona Española, a la vez que propagaba la vacunación en la metrópoli, organizó una expedición sanitaria con objeto de que las poblaciones de las entonces colonias españolas en América y Filipinas se beneficiaran del gran descubrimiento. En la decisión de promover esta magna expedición jugó un papel importante una circunstancia personal del monarca

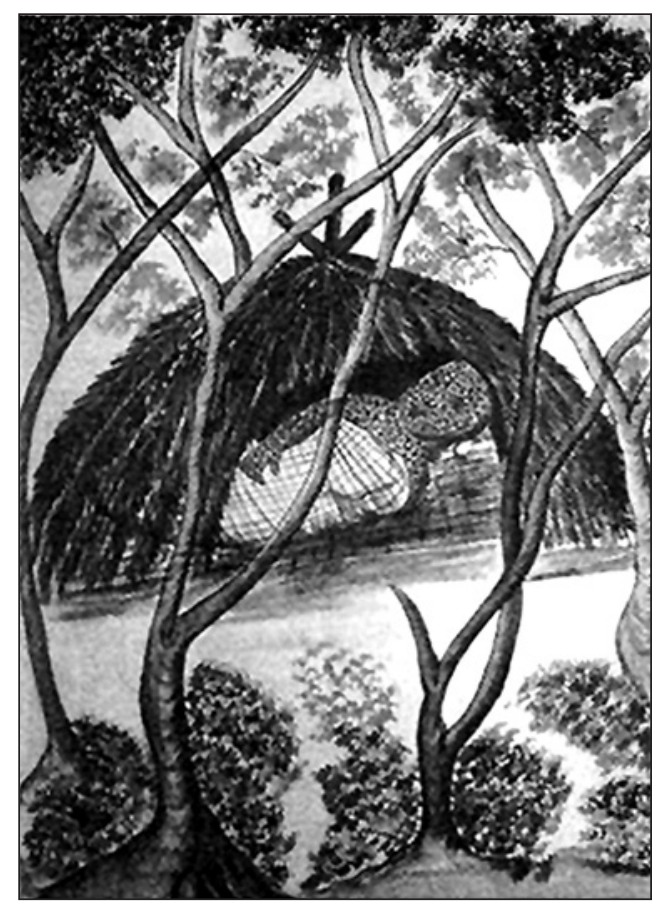

Figura 1. "Indio con viruela". Lámina de la obra del obispo peruano Baltázar Jaime Martínez Campañón, Trujillo del Perú. 1786. (Ed. de Madrid. Ed. Cultura Hispánica del Centro Iberoamericano de Estudios).
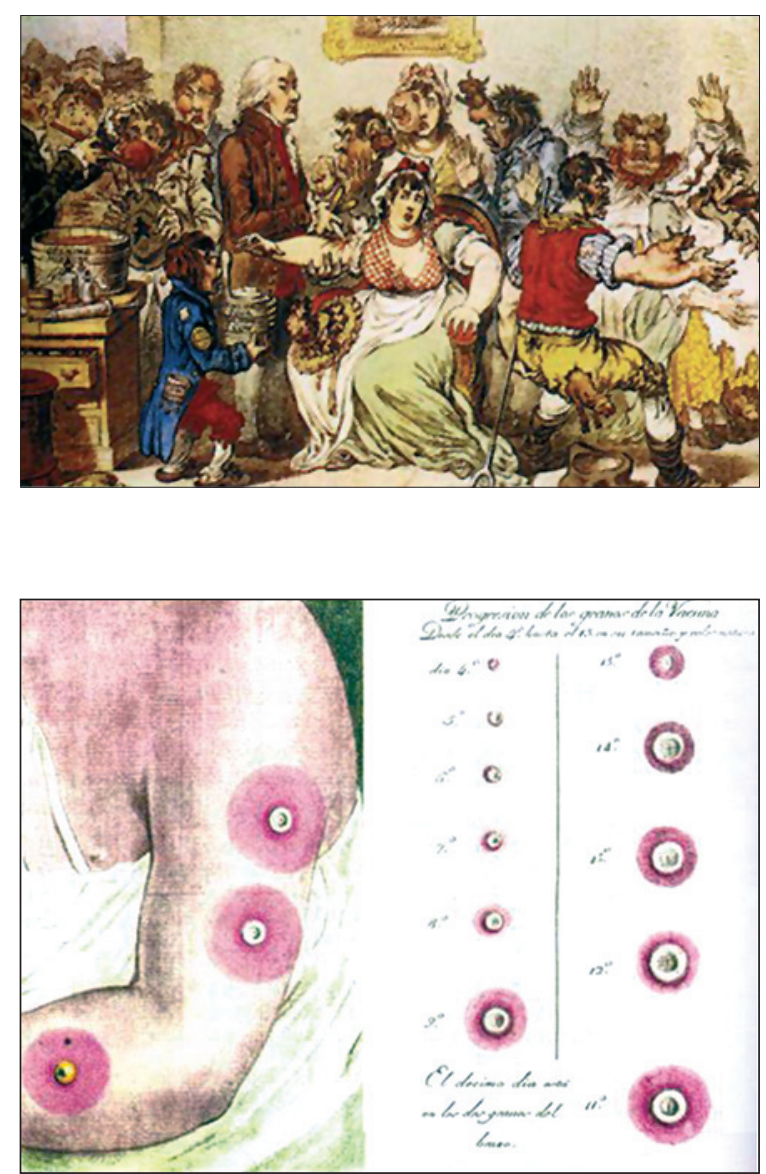

Figura 2. Caricatura de los supuestos efectos de la vacuna dentro de la campaña contra la vacuna
Figura 3. Diferentes estadíos en la evolución de los cambios experimentados en la superficie cutánea de las personas vacunadas. Grabados del artista valenciano Juan Ximeno Carrero. Biblioteca y Museo histórico-médicos. Valencia. 
Figura 4. El rey Carlos IV (del cuadro de Goya "La familia de Carlos IV).
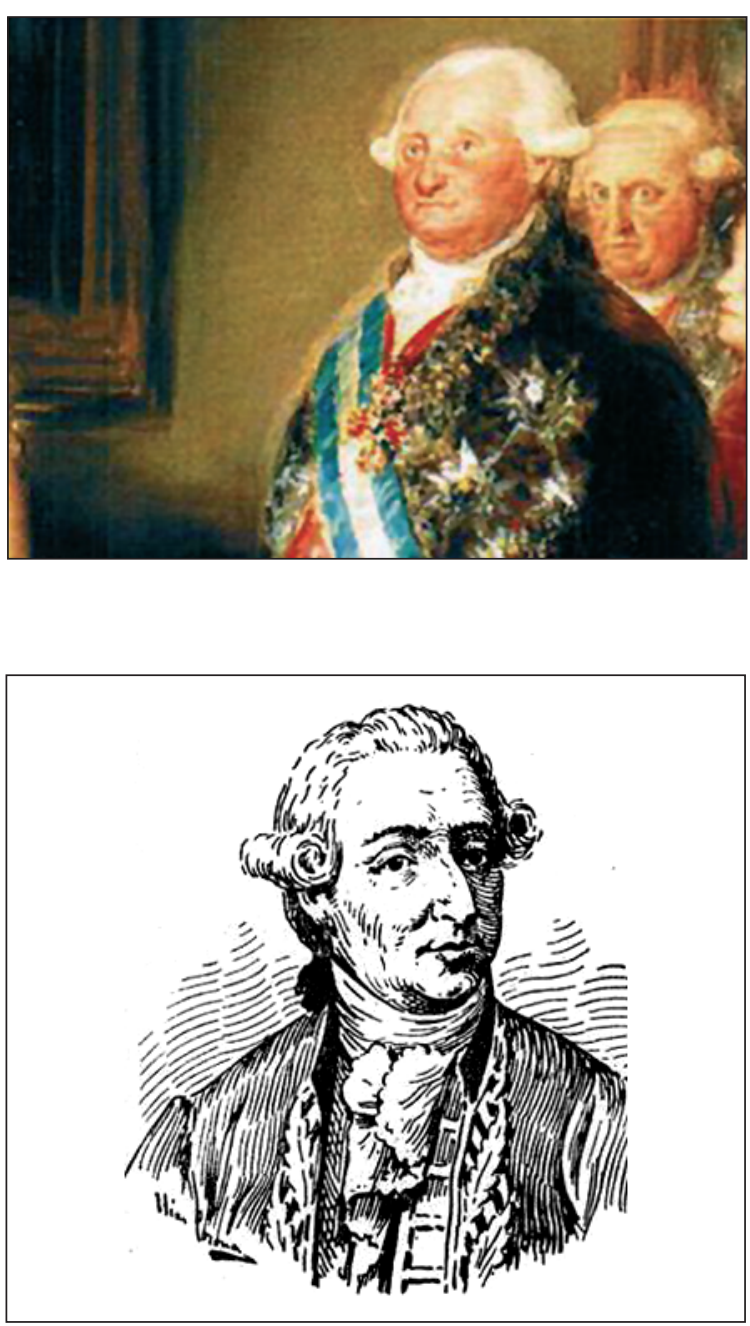

Figura 5. Francisco Xavier de Balmis. Grabado de Elías Corona.
(Figura 4): una hija del rey Carlos IV había sido víctima de la viruela y se propuso evitar tan amarga experiencia al mayor número posible de sus súbditos en todos los continentes. Los objetivos que se pretendían cumplir con esta empresa eran:

- Difundir la vacuna desde el Reino de España a todos los Virreinatos ultramarinos.

- Instruir a los sanitarios locales de las poblaciones visitadas para dar continuidad a la práctica de la vacunación a lo largo del tiempo.

- Crear en los virreinatos "Juntas de Vacunación" como centros para conservar, producir y abastecer de vacunas activas para mantener la campaña de forma permanente.

Para cumplir estos objetivos la expedición de la vacuna empezó a prepararse en marzo de 1803. Como sistema de financiación se estableció que los gastos de navegación hasta las costas de ultramar corrieran a cargo de la Real Hacienda. Una vez allí los gastos de las expediciones locales y de la creación de las Juntas de Vacunación serían sufragados por los Tributos de Indios, Censos de Indios y Diezmos Eclesiásticos, que eran los procedimientos habituales para mantener las arcas de la Hacienda Pública en los Virreinatos.

El seis de Junio de este año se publicó una Real Orden en la que se disponía su organización y se nombraba director a Xavier de Balmis (Figura 5) quien quedó encargado de comprar todo lo necesario para el buen éxito de la empresa. El nombramiento de Xavier de Balmis como jefe de la expedición no fue casual ya que difícilmente pudo encontrarse otra persona en quien concurrieran tantas cualidades y preparación óptimas para esta misión. Nacido en Alicante, tenía una sólida formación intelectual, talante optimista y espíritu muy activo, poseía dotes de mando y afrontaba los peligros con valentía mirando siempre el bien de los demás. Cirujano militar, intervino en la introducción en España de la materia médica procedente de América, continente que había visitado en varias ocasiones. Cuando se hizo cargo de la expedición llevaba ya muchos años dedicado a la sanidad. Fue también un decido partidario de la variolización, método con el que había adquirido una amplia experiencia. Otra obra de Balmis que demuestra su interés por la vacuna fue la traducción del francés en marzo de 1803 del libro de Jacques Louis Moreau de la Sarthe Tratado histórico y práctico de la Vacuna. Este libro sirvió de guía general de la vacunación y entre el material cargado en la expedición figuraban un buen número de ejemplares para su distribución en las distintas Juntas de Vacunación que fueron organizándose por tierras americanas.

Como subdirector de la expedición fue nombrado Josep Salvany de un carácter muy diferente al de Balmis ya que, si bien compartía con él el entusiasmo por la empresa,

Figura 6. La goleta "María Pita". 
carecía de sus dotes organizadoras. Mientras que Balmis era muy crítico con sus subordinados, Salvany tenía de todos ellos una magnífica opinión. Ayudantes de Balmis y Salvany fueron los médicos Manuel Julián Grajales y Antonio Gutiérrez Robredo y los practicantes Francisco Pastor Balmis y Rafael Lozano Pérez. A ellos se añadieron los enfermeros Basilio Bolaños, Pedro Ortiga y Antonio Pastor. El barco contratado para la expedición fue la corbeta María Pita (Figura 6) teniendo como capitán a Pedro del Barco. La única mujer de la expedición fue Isabel Sendales, rectora de la casa de expósitos de A Coruña que desempeñó el papel de figura maternal para los niños reclutados.

Naturalmente faltaba el elemento fundamental y la razón de ser de la expedición: la vacuna. Los viajes transatlánticos duraban como mínimo un mes, en consecuencia una persona vacunada al partir ya habría desarrollado toda la enfermedad durante la travesía y no serviría como fuente para obtener linfa vacunal. Por esta razón se reclutaron niños para irlos vacunando progresivamente. Al partir fueron vacunados dos niños que a lo largo de una semana desarrollaron las vesículas características de la viruela vacuna. De estas vesículas frescas se obtuvo linfa con la que se vacunaron otros dos niños y así sucesivamente. Las linfas de las vesículas más frescas se conservaban al vacío en ampollas de cristal especiales. De esta forma se conseguía llegar a las distintas etapas de la expedición con vacunas en perfecto estado.

Los niños actuaron como auténticos reservorios naturales de la vacuna que garantizaban el transporte de tan precioso material biológico en perfectas condiciones. No fue fácil reclutar suficiente cantidad de niños tanto para el grupo inicial que se embarcó en A Coruña como para los grupos que se fueron creando en las distintas etapas en tierras americanas. Las condiciones requeridas eran que estuvieran sanos, que tuvieran edades entre 8 y 10 años y que no hubieran pasado la viruela "natural" ni estuvieran ya vacunados. A estos niños se les prometía alimentación, vestido y cuidados a cargo del erario público y una buena educación hasta que pudieran desempeñar una profesión digna. A pesar de lo atractivo de la oferta pocas madres se mostraron dispuestas a ceder sus hijos a esta aventura por lo que hubo que recurrir a niños expósitos, abandonados, hijos de madres solteras o de familias desestructuradas.

El grupo inicial de niños que se embarcó estuvo formado por cuatro niños de los hospicios de Madrid y 18 de los orfanatos de Santiago de Compostela haciendo un total de 22 niños pronto conocidos como "galleguitos" (Figura 7). Este número era ligeramente inferior a los 25 que había calculado Balmis para el éxito de la misión. Entre estos niños estaba el hijo de la regidora de un orfanato de Santiago Isabel Sendales, la citada única mujer en la expedición.

Tras febriles trabajos, el 30 de Noviembre partía ya del puerto de A Coruña la "María Pita" iniciándose así la expedición filantrópica de la vacuna que había de durar hasta el 14 de agosto de 1806 después de haber dado la vuelta al mundo (Figura 8). En el equipaje Balmis incluyó

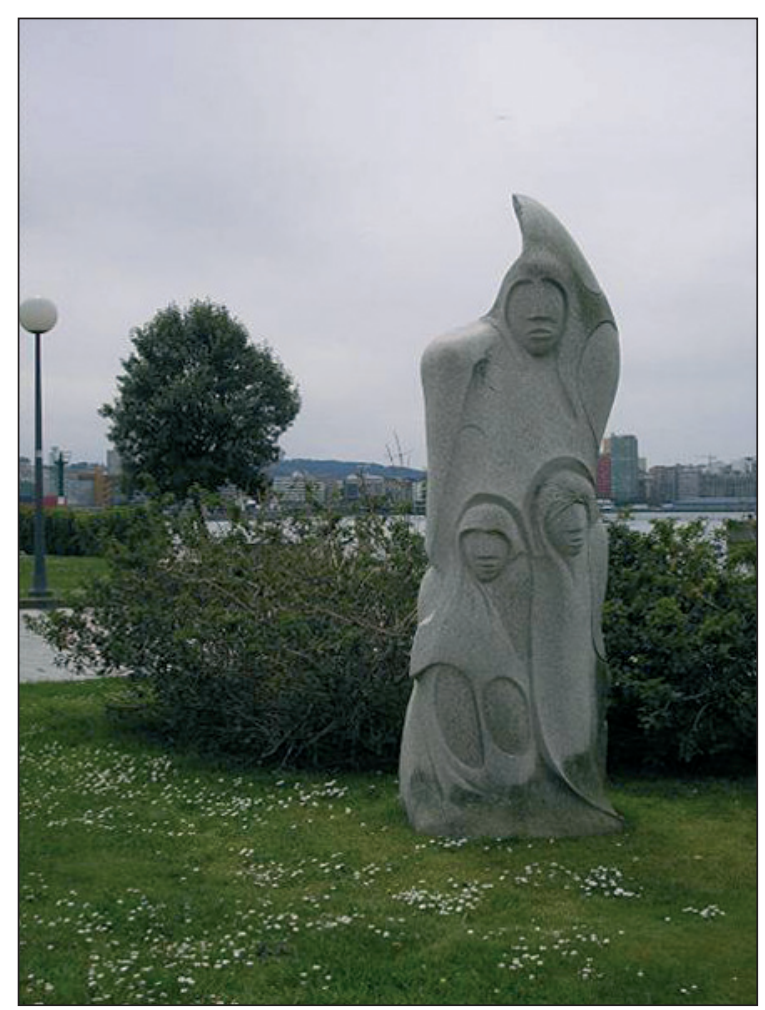

Figura 7. Monumento a los "galleguitos" en el puerto de A Coruña

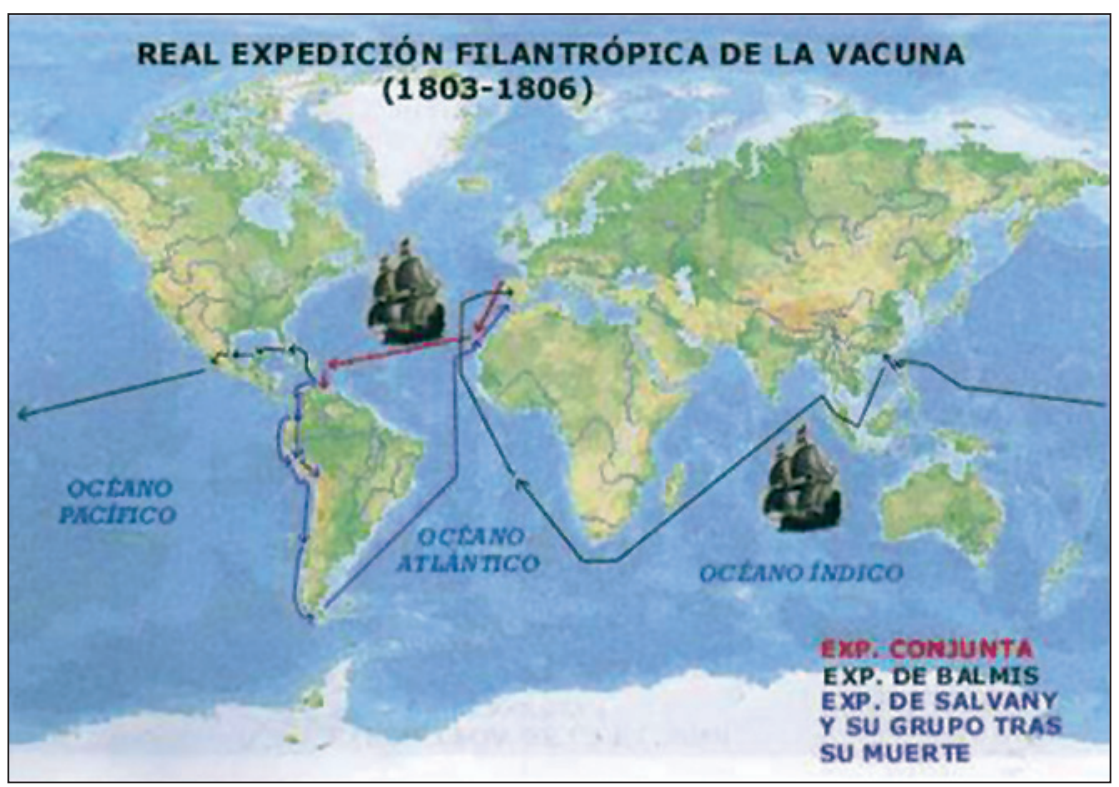

Figura 8. Itinerario de la expedición de Balmis. 
Figura 9. Monumento de Balmis en Alicante.
500 ejemplares del mencionado libro de Moreau de la Sarthe, termómetros y barómetros meteorológicos, así como varios miles de ampollas de cristal donde conservar una gota de linfa vacunal. Estas ampollas se cerraban con
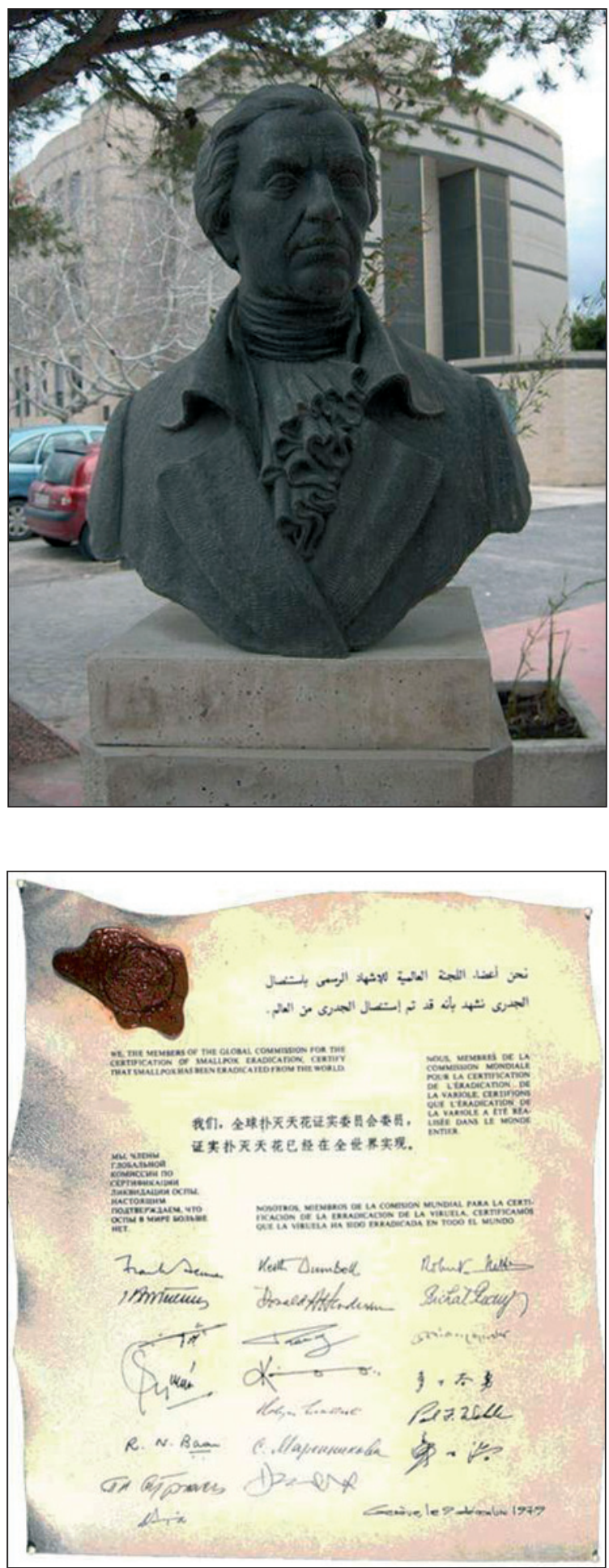

parafina después de haber hecho el vacío para lo cual también se dispuso de varias máquinas neumáticas.

La primera etapa fue Santa Cruz de Tenerife donde permaneció un mes para vacunar a la población de aquella ciudad y establecer un centro de vacunación desde donde difundir la vacuna al resto de las Islas Canarias. La siguiente etapa fue Puerto Rico donde las autoridades no prestaron atención ni colaboraron con los expedicionarios ya que allí ya se había difundido la vacuna desde las colonias inglesas y no se encontraron niños en condiciones de dar continuidad a los pases vacunales. A continuación, después de una tormentosa travesía, llegaron al puerto venezolano de Puerto Cabello, en lugar de hacerlo como estaba previsto al de La Guayra, y desde allí por tierra hasta Caracas donde se les dispensó una cordial acogida y crean la primera Junta de Vacuna del continente desde donde se difundió la vacuna a toda Venezuela. Esta Junta de Vacuna sirvió de modelo para las muchas que se instauraron luego a lo largo de la expedición.

En Caracas la expedición se dividió en dos grupos, uno dirigido por Salvany se dirigiría por tierra hasta Santa Fe y desde allí a muchos otros territorios de América del Sur, y otro bajo la dirección del propio Balmis, lo haría por mar, nuevamente a bordo de la corbeta María Pita. Se dirigió a La Habana, donde se encontró también con que ya se había introducido la vacuna. Ante la dificultad de encontrar allí niños aptos para la expedición Balmis logra la contratación de tres esclavas cubanas como portadoras de la vacuna. De esta forma llegan al puerto de Sisal en la península mexicana de Yucatán y desde allí a Mérida donde establecen una nueva Junta de Vacuna desde donde la extienden por todo el Virreinato de Nueva España y territorios limítrofes incluyendo la Capitanía General de Guatemala. De regreso a Sisal ponen rumbo a Veracruz donde se encuentran nuevamente con la dificultad de encontrar niños aptos y en esta ocasión recurren a la tropa llegando así por tierra a la capital del Virreinato, la ciudad de México. En tierras mexicanas se establecieron varias Juntas de Vacunas desde las que se difundió por todo el amplio territorio del Virreinato.

Desde el puerto de Acapulco la expedición se embarcó en el navío Santa Bárbara que cubría la línea regular a Manila y donde los 26 niños mexicanos que se lograron reclutar padecieron unas condiciones mucho peores de los que se embarcaron en la María Pita. En Manila se estableció nuevamente una Junta de Vacuna como centro de difusión de la vacuna en las Islas Filipinas. Desde allí se dirigió al enclave portugués de Macao en la costa china. La travesía, realizada a bordo de la fragata Diligencia fue durísima por sorprenderles un violento tifón. A partir de Macao se realizan diversas expediciones en territorio chino divulgando la vacuna. Balmis está extenuado y se siente enfermo por lo que se ve obligado a ceder la dirección de la expedición a su ayudante Antonio Gutiérrez
Figura 10. Cerificado de la OMS de la erradicación de la viruela en todo el mundo. 
y decidió regresar a Europa a bordo del navío portugués Bon Jesús de Alem con destino Lisboa. En una escala en la isla inglesa de Santa Elena, en pleno Atlántico, Balmis, que todavía conservaba una reserva de vacuna, logró convencer al gobernador de su conveniencia y utilidad, cosa facilitada por la entrega que le hizo éste de un paquete sin abrir desde hacía años donde figuraba una cantidad de linfa vacunal y un escrito de puño y letra del propio Edward Jenner con instrucciones para su aplicación.

Llegado a Lisboa Balmis se dirigió en carruaje a Madrid donde el rey Carlos IV le recibió con todos los honores el 7 de septiembre de 1806. Balmis murió en Madrid 1819 (Figura 9). Por su parte Salvany, que había realizado en tierras sudamericanas unas campañas de vacunación parangonables con las de Balmis, había fallecido en Cochabamba en 1810.

Se daba así por terminada esta fabulosa expedición que permitió hacer llegar a miles, tal vez millones de seres humanos un avance tan trascendental como la vacuna antivariólica que les permitió librarse de uno de los grandes azotes que ha sufrido la humanidad elogiada por el propio Jenner. Se había dado así un paso decisivo para que, en 1980 la Organización Mundial de la Salud declarara la viruela erradicada del planeta Tierra (Figura 10).

Tal vez esta expedición no haya sido reflejada en la literatura, en el cine o en la televisión en la medida que se merece, sobre todo si se compara con otras hazañas que han tenido mucho menor trascendencia para la humanidad. En cualquier caso no han dejado de haber escritores que han utilizado el tema tanto para el teatro, como el venezolano Andrés Bello en su obra "Venezuela consolada" (1804) como para la narrativa, como la norteamericana de origen dominicano Julia Alvarez en su novela "Saving the World" (2006) traducida al español con el título de "Para salvar el mundo".

Sea este relato un modesto homenaje a aquellos auténticos héroes que entregaron todo su saber y todos sus esfuerzos a favor de la humanidad y en particular de los pueblos latinoamericanos, sin olvidar a aquellos niños que consagraron los mejores años de su infancia a tan noble objetivo.

Agradecimientos. Quiero agradecer muy cordialmente a la profesora Susana María Ramírez Martín, autora del libro "La salud del Imperio", y a los profesores Emili Balaguer y Rosa Ballester, autores del libro "La Real Expedición Filantrópica de la Vacuna(1803-1806)” por el apoyo prestado.

\section{Resumen}

Seis años después de Jenner descubriera la vacuna antivariólica, el rey Carlos IV dispuso emprender campañas vacunales en todos sus reinos. La campaña ultramarina fue dirigida por el médico militar valenciano Xavier de Balmis ayudado por el catalán Josep Salvany. El trasporte de la vacuna a América se realizó 'in vivo’ en 22 niños. La expedición partió de A Coruña en noviembre de 1806 y llegó al puerto venezolano de La Guayra donde se dividió en dos: Una, al mando de Josep Salvany, siguió por tierra a Colombia, Ecuador, Perú y Bolivia. Salvany falleció en la ciudad boliviana de Cochabamba. La otra, al mando del propio Balmis, siguió por mar a Cuba y México. Desde Acapulco llegó a Manila y de allí a Macau desde donde se adentró en las zonas chinas adyacentes introduciendo así la vacuna en el continente asiático. 